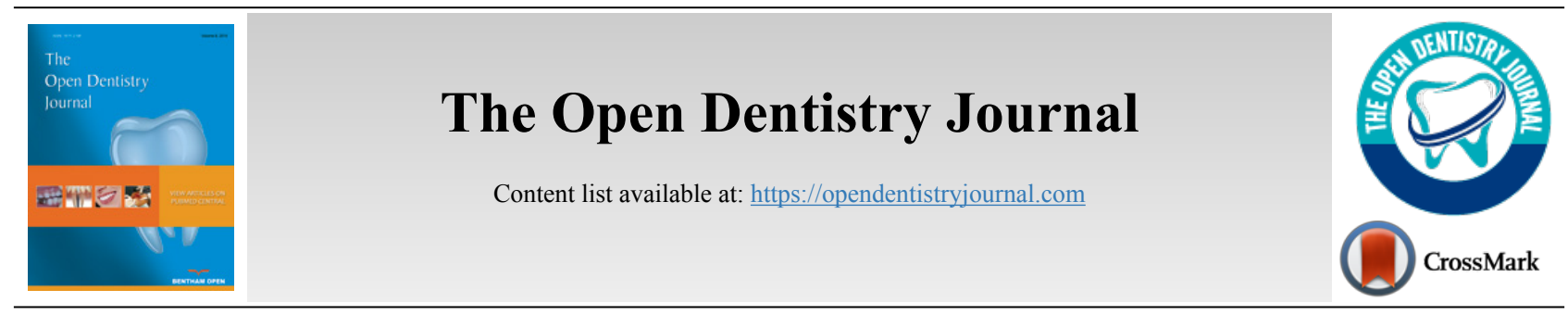

\title{
CORRIGENDUM
}

\section{Mineralized Plasmatic Matrix for Horizontal Ridge Augmentation in Anterior Maxilla with and without a Covering Collagen Membrane}

\author{
Ehab Abdelfadil ${ }^{1}$ and Wael Aboelmaaty ${ }^{2,3, *}$ \\ ${ }^{\prime}$ Department of Oral and Maxillofacial Surgery, Faculty of Dentistry, Mansoura University, Mansoura, Egypt \\ ${ }^{2}$ Department of Oral Radiology and Diagnostic Sciences, Faculty of Dentistry, Mansoura University, Mansoura, Egypt \\ ${ }^{3}$ Depatment of Oral Radiology, College of Dentistry, King Saud bin Abdulaziz University of Health Sciences, Riyadh, Saudi Arabia
}

We would like to apologize for the errors that occurred in the online version of the article. Duplicate paragraphs in abstract and incorrect figures 1 and 2 have been published in the article entitled "Mineralized Plasmatic Matrix for Horizontal Ridge Augmentation in Anterior Maxilla with and without a Covering Collagen Membrane" in "The Open Dentistry Journal”, 2020 Dec 31;14(1) [ 1].

The original article can be found online at https://opendentistryjournal.com/VOLUME/14/PAGE/743/FU LLTEXT/

\section{Original:}

\section{Materials and Methods:}

Sixteen edentulous spaces were randomly divided into 2 equal groups. MPM was used for horizontal ridge augmentation with and without a covering collagen membrane (group 1 and 2, respectively). Cone Beam CT images were obtained preoperatively as well as 1 week and 4 months postoperatively to evaluate alveolar ridge and the resorption of the grafting material at 3 predetermined points along the site where the future dental implant will be placed.

Student's t-test (Unpaired) was used for comparing two different groups with quantitative parametric data and student's t-test (Paired) was used for comparing two related groups with quantitative parametric data while repeated measures ANOVA (Analysis of variance) followed by post-hoc Bonferroni was used for comparing more than two related groups with quantitative parametric data.

Student's t-test (Unpaired) was used for comparing two different groups with quantitative parametric data and student's t-test (Paired) was used for comparing two related groups with quantitative parametric data while repeated measures ANOVA (Analysis of variance) followed by post-hoc Bonferroni was used for comparing more than two related groups with quantitative parametric data.

\section{Corrected:}

\section{Materials and Methods:}

Sixteen edentulous spaces were randomly divided into 2 equal groups. MPM was used for horizontal ridge augmentation with and without a covering collagen membrane (group 1 and 2, respectively). Cone Beam CT images were obtained preoperatively as well as 1 week and 4 months postoperatively to evaluate alveolar ridge and the resorption of the grafting material at 3 predetermined points along the site where the future dental implant will be placed.

Student's t-test (Unpaired) was used for comparing two different groups with quantitative parametric data and student's t-test (Paired) was used for comparing two related groups with quantitative parametric data while repeated measures ANOVA (Analysis of variance) followed by post-hoc Bonferroni was used for comparing more than two related groups with quantitative parametric data. 


\section{Original:}

Figs. 1 and 2 were:
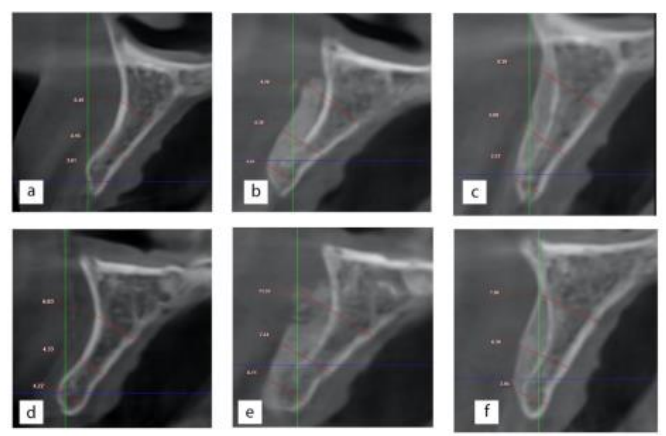

Fig. (1). Radiographic evaluation. Preoperative bone width for group $1 \& 2$ (a \& d). Postoperative ridge measurement for group $1 \& 2$ (b \& e). Final bone width after 4 months for group $1 \& 2$ (c \& f).
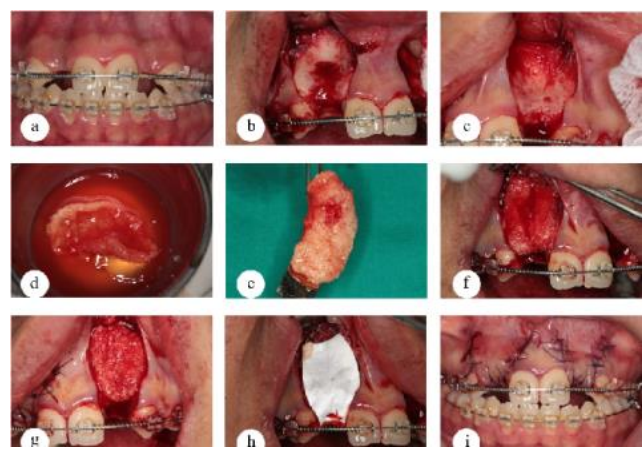

Fig. (2). Surgical procedures for horizontal ridge augmentation using MPM. (a) preoperative photograph. (b \& c) the deficient alveolar ridge after flap elevation. ( $\mathrm{d} \& \mathrm{e}$ ) preparation of MPM. (f \& g) MPM placed over the labial bone for group 1 and 2 respectively. (h) a collagen membrane used to cover MPM in group 1. (i) flap repositioning and suturing.

\section{Corrected:}

Figs. 1 and 2 have been revised as:
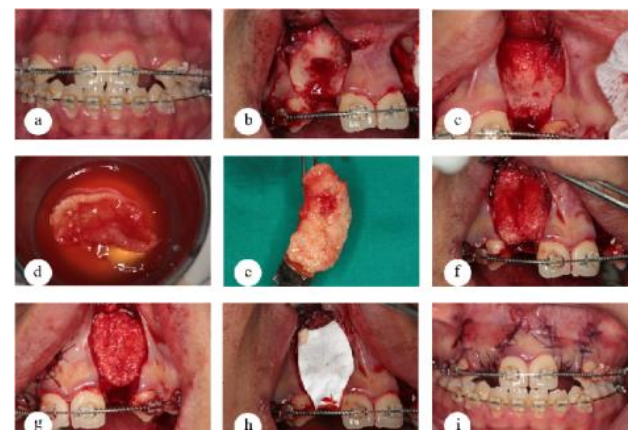

Fig. (1). Surgical procedures for horizontal ridge augmentation using MPM. (a) preoperative photograph. (b \& c) the deficient alveolar ridge after flap elevation. ( $\mathrm{d} \&$ e) preparation of MPM. (f \& g) MPM placed over the labial bone for group 1 and 2 respectively. (h) a collagen membrane used to cover MPM in group 1. (i) flap repositioning and suturing. 

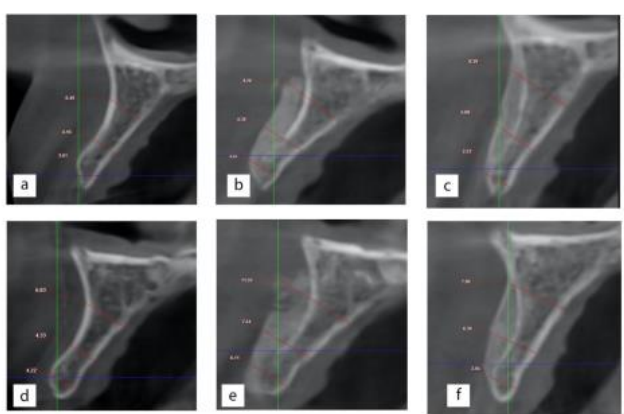

Fig. (2). Radiographic evaluation. Preoperative bone width for group $1 \& 2$ (a \& d). Postoperative ridge measurement for group $1 \& 2$ (b \& e). Final bone width after 4 months for group $1 \& 2$ (c \& f).

\section{REFERENCE}

[1] Abdelfadil E, Aboelmaaty W. Mineralized Plasmatic Matrix for
Horizontal Ridge Augmentation in Anterior Maxilla with and without a Covering Collagen Membrane. Open Dent J 2020; 14(1) [http://dx.doi.org/10.2174/1874210602014010743]

\section{2021 Abdelfadil and Aboelmaaty}

This is an open access article distributed under the terms of the Creative Commons Attribution 4.0 International Public License (CC-BY 4.0), a copy of which is available at: (https://creativecommons.org/licenses/by/4.0/legalcode). This license permits unrestricted use, distribution, and reproduction in any medium, provided the original author and source are credited. 